\title{
Clinical effects of repetitive transcranial magnetic stimulation combined with atomoxetine in the treatment of attention-deficit hyperactivity disorder
}

This article was published in the following Dove Press journal:

Neuropsychiatric Disease and Treatment

Pengfei Cao, ${ }^{1,2, *}$ Jun Xing, ${ }^{1, *}$

Yin Cao, ${ }^{3}$ Qi Cheng,' Xiaojing Sun,' Qi Kang,' Libin Dai,' Xianju Zhou, ${ }^{3}$ Zixiang Song'

'Psychological Centre for Adolescents and Children, The Affiliated $102^{\text {nd }}$ Hospital of The Second Military Medical University of People's Liberation Army of China, Shanghai, China; ${ }^{2}$ Department of Political Affairs, College of Politics, National Defense University of People's Liberation Army of China, Beijing, China; ' ${ }^{2}$ aboratory of Neurological Diseases, Department of Neurology, The Affiliated Changzhou No 2 People's Hospital of Nanjing Medical University, Changzhou, China

*These authors contributed equally to this work

Correspondence: Xianju Zhou Laboratory of Neurological Diseases, Department of Neurology, The Affiliated Changzhou No 2 People's Hospital of Nanjing Medical University, 29 Xinglong Alley, Changzhou, Jiangsu Province 213003, China

Tel +86 I35 85333708

Email xianju_zhou@yahoo.com

Zixiang Song

Psychological Centre for Adolescents and Children, The Affiliated $102^{\text {nd }}$ Hospital of the Second Military Medical University of PLA, 55 North Heping Road, Changzhou, Jiangsu Province 213003, China

Tel +86 I33 27895177

Email axiang952@sohu.com
Objective: To explore the effect of repetitive transcranial magnetic stimulation (rTMS) combined with atomoxetine (ATX) in the treatment of attention-deficit hyperactivity disorder (ADHD).

Methods: Sixty-four patients with newly diagnosed ADHD were enrolled from January 2016 to October 2017 from Psychological Centre for Adolescents and Children at 102th Hospital of People's Liberation Army of China. These patients were randomly assigned to three groups according to treatment method: the rTMS group, the ATX group, and the rTMS+ ATX group. Before treatment and 6 weeks after treatment, clinical symptoms and executive functions of ADHD patients were evaluated with the Swanson, Nolan, and Pelham, Version IV (SNAP-IV) Questionnaire, continuous performance test, three subtests (arithmetic, digit span, and coding) of Wechsler Intelligence Scale for Children, as well as Iowa Gambling Tasks (IGT). The effects of treatment were compared among three groups.

Results: After 6 weeks of treatment, the scores of all factors in the SNAP-IV questionnaire were lower than those before treatment in the three groups; the scores of three subtests of Wechsler Intelligence Scale for Children, continuous performance test, and IGT were also significantly higher than those before treatment. The rTMS+ ATX group had a better improvement in attention deficits and hyperactivity impulse on the SNAP-IV questionnaire compared with the other groups, and also had a higher efficacy on cold and hot executive functions such as arithmetic, forward numbers, coding, and IGT. In addition, the ATX group performed better than the rTMS group in coding and IGT. Conclusion: rTMS, ATX, and the combination therapy are effective in improving core symptoms and executive function in patients with ADHD. The combined treatment has significant therapeutic advantages over the single treatment groups. Compared with rTMS, the drug therapy has a better improvement in coding and IGT.

Keywords: attention-deficit hyperactivity disorder, repetitive transcranial magnetic stimulation, atomoxetine, executive function

\section{Introduction}

Attention-deficit hyperactivity disorder (ADHD) is a common neuropsychiatric disorder in children. Its core symptoms are inattention and hyperactivity, which seriously affect the cognitive, behavioral, and emotional aspects of the patient. The prevalence of ADHD in children is 5\%, and the ratio between males and females is about $2: 1{ }^{1}$ The pathogenesis of ADHD has not yet been clarified. Currently, it is widely believed that it is caused by the synergistic actions of various biological-psychological-social factors. 
Its pathogenesis and development are closely related to many factors such as heredity, neural development, family, and society. ${ }^{2}$

ADHD is thought to be a polygenic genetic disease associated with some neurotransmitter-related genes, such as dopamine (DA), 5-hydroxytryptamine, norepinephrine (NE), as well as their metabolite-related genes. The dysfunction of these neurotransmitters is thought to be the main cause of ADHD. ${ }^{3,4}$ Functional magnetic resonance imaging (fMRI) and positron emission imaging (PET) revealed that the occurrence of ADHD is related to anatomic defects and functional changes in the prefrontal lobe, temporal lobe, basal ganglion, corpus callosum, and the cerebellum of the patients. ${ }^{5,6}$ Further, fMRI has demonstrated consistent differences in frontostriatal functioning, specifically, right dorsolateral prefrontal cortical (rDLPFC) functioning in ADHD children when compared with healthy controls. ${ }^{7}$ Moreover, cortical maturation was delayed by about 3 years in ADHD children, most prominently in prefrontal regions. ${ }^{8}$ Studies have shown decreased frontocerebral metabolism in ADHD adults using PET, xenon inhalation, and SinglePhoton Emission Computed Tomography. ${ }^{9,10}$ In addition, parental discord or divorce, poor parent-child relationships, poor family education, negative childhood experiences, the effects from bad social habits, mother smoking and alcohol consumption and substance abuse during pregnancy, premature delivery, low birth weight, and intracranial infection are also risk factors for ADHD children. ${ }^{11,12}$

The treatment of ADHD mainly includes drug therapy, physical therapy (eg, repetitive transcranial magnetic stimulation [rTMS]), and cognitive behavioral intervention. ${ }^{13}$ Atomoxetine (ATX), as a representative nonstimulant, has therapeutic effects on ADHD like methylphenidate (a stimulant), ${ }^{14}$ but ATX only increases the level of noradrenaline (but not DA) in the subcortical region. Nor does ATX affect DA concentrations in the striatum and limbic systems. Moreover, ATX significantly reduces the risk of drug abuse, and is relatively safe and tolerable in adolescents and children. ${ }^{15-17}$ rTMS delivers a magnetic field on a coil through strong current and acts on a specific cortex of the brain to achieve reconstruction of the cortical functions. It is a noninvasive and painless neurophysiological technique that regulates cerebral cortical function. ${ }^{18}$ As a physical therapy method, rTMS has significant therapeutic effects on anxiety disorders, schizophrenia, depression, and other mental diseases. ${ }^{19-21}$ Its most prominent advantages are safety and minimal side effects. ${ }^{22}$ Some studies have reported that rTMS can promote the secretion of DA by stimulating the prefrontal lobe of the brain, thereby improving the symptoms of ADHD. ${ }^{23}$

Both rTMS and ATX can effectively improve attention deficits, hyperactivity, and other symptoms in ADHD patients. However, there is no report on the effect of combination therapy (rTMS+ ATX) in the treatment of ADHD. Thus, the purpose of this study was to compare the efficacy of the three methods in the treatment of ADHD, and to observe whether the rTMS combined with ATX has a certain advantage over rTMS or ATX.

\section{Materials and methods Research subjects ADHD group}

Sixty-four patients were newly diagnosed with ADHD from January 2016 to October 2017 in the Psychological Center for Adolescents and Children at the 102nd Hospital of People's Liberation Army (PLA). All were Han people and righthanded, including 54 males and 10 females, ranging from 6 to 13 years old and with an average age of $8.53 \pm 2.30$ years. All the enrolled patients were initially diagnosed by two pediatric psychiatry attending physicians and were eventually diagnosed by a chief physician.

The enrollment criteria were as follows: 1) met the diagnostic criteria for ADHD in the fifth edition of Diagnostic and Statistical Manual of Mental Disorders (DSM-5) of the American Psychiatric Association; 2) no history of other mental disorders (such as mental retardation) and organic brain diseases that affected cognitive functions; 3 ) no severe systemic diseases including heart, liver, lung, and kidney diseases; 4) IQ $\geq 80$ by Wechsler Intelligence Scale for Children (WISC). Exclusion criteria were as follows: 1) other drugs or treatments in addition to the treatment used in this study; 2) treated with TMS or electroconvulsive therapy; 3) taking or having taken monoamine oxidase inhibitors within 14 days; 4) a history of epilepsy, brain trauma, or brain surgery; 5) rTMS contradictions such as brain metal device implants. ${ }^{24}$ This study was approved by the Ethics Committee of 102nd Hospital of PLA. All the enrolled patients and parents (guardians) signed the informed consent. This study was carried out in accordance with the principles of the Declaration of Helsinki.

\section{Treatment group}

Sixty-four patients with ADHD were randomly assigned to three groups according to a random distribution table: the rTMS group, the ATX group, and the rTMS+ ATX group. The total course of the treatment was 6 weeks. 
The rTMS group: in this study, Magneuro100 magnetic field stimulator (VCB001, Nanjing Weisi Medical Technology Co., LTD) with a figure-of-eight coil was used. rTMS treatment parameter settings based on one previous study: $10 \mathrm{~Hz}, 4$ seconds stimulation, 26 seconds interval, $100 \%$ intensity of resting motor threshold (rMT), 2,000 pulses per session, a total of 25 minutes/session, one session/day, five sessions/week (from Monday to Friday), a total of 6 weeks of treatment (ie, 30 sessions). ${ }^{25}$ Stimulus intensity was obtained according to rMT for each individual, which was defined as the lowest intensity to elicit at least 5 out of 10 electromyography with amplitude $>50 \mu \mathrm{V}$ peak-topeak in the first dorsal interosseous muscle of the left hand. Stimulation site: rDLPFC, located at the site where the coil stays at the MT detection, then moved forward by $5 \mathrm{~cm}$ in parallel. The coil was placed tangentially toward the scalp over rDLPFC in a frontal-posterior orientation.

The ATX group: atomoxetine hydrochloride capsules (Strattera, Eli Lilly and Company, Indianapolis, IN, USA) were used in this study. Initial oral ATX dose was $0.5 \mathrm{mg} /(\mathrm{kg} \cdot$ day), and 3 days later, it was increased to $1.2 \mathrm{mg} /(\mathrm{kg} \cdot \mathrm{day})$. ATX was administered once daily after breakfast for 6 weeks. ${ }^{16}$

The rTMS+ ATX group: The same rTMS stimulation parameters and the same ATX dose were used in the combination therapy for 6 weeks.

\section{Clinical symptom assessment for ADHD}

The SNAP-IV questionnaire was used to assess the core symptoms of ADHD before treatment and 6 weeks after treatment. In order to maintain consistency of the evaluation criteria, the assessment was completed by the same experienced researcher before and after treatment. The researcher was blinded to the treatment. The scores before and after treatment were compared to evaluate the improvement of clinical symptoms. And the therapeutic effects among the three groups were also compared.

The SNAP scale is based on the diagnostic criteria of ADHD in DSM. ${ }^{26,27}$ Its reliability and validity are in accordance with the psychometric standard. The scale has been widely used in clinical practice and scientific research as an important tool of ADHD in screening, auxiliary diagnosis, therapeutic efficacy, and symptom improvement evaluation. ${ }^{28}$ Twenty-six items are included and summarized into three factors: attention deficit, hyperactivity impulse, and oppositional defiance. According to the severity of the symptoms, parents checked 0-3 scale based on their general impressions on their children. Mean score of 1 was defined as the demarcation for attention deficit and hyperactivity impulsivity;
Mean score $\leq 1$ indicated "normal" or "remission"; mean score $\geq 2$ indicated "abnormal". For oppositional defiant disorder, four or more items with a score of 2 or 3 indicated "abnormal".

\section{Executive function test}

Executive function refers to the ability to coordinate various cognitive processes when completing complex cognitive tasks. In the core symptoms of ADHD, attention deficit is related to "cold" executive function, while hyperactivity impulse is related to "hot" executive function. ${ }^{29}$ "Hot" executive function, relative to "cold" executive function, has extensive emotional involvement, which is related to adventurous emotional decision-making or flexible assessment of the emotional responsiveness to thrill, making emotional and motivational problems solved. ${ }^{30}$ The "hot" executive function is mainly associated with the cerebral orbital and medial prefrontal cortex. Injury at these sites may lead to impediment in impulse control, behavioral regulation, and mood. ${ }^{31}$ Therefore hyperactivity impulse symptoms of ADHD are more closely related to the "hot" executive function. ${ }^{32}$

\section{"Cold" executive function test}

Arithmetic, digit span, and coding, which are the three subtests of the continuous performance test (CPT) and the WISC, were conducted before treatment and 6 weeks after treatment. This study followed the protocol of CPT (Chinese version, translated by the Institute of Mental Health at the Peking University) in 2004 MATRICS Consensus Cognitive Battery by the National Institute of Health..$^{33}$ These subtests were set up at three levels of difficulty, which are two-, three- and four-digit numbers in turn. Each subtest contains 150 strings of numbers. Participants were required to make response when two adjacent strings of numbers were same. The main indicators examined were hits for the pairs with same numbers, almost identical consecutive numbers (false alarms), random number of answers (randoms), and the total score of each subtest (DEPRIME).

\section{WISC test (arithmetic, digit span, and coding)}

WISC is a test tool developed by Wechsler to assess the intelligence of children aged from 6 to 16 years. In this study, three subtests (including arithmetic and digit span from the verbal subscale, and coding in the performance subscale) were used to examine the "cold" executive function of ADHD patients..$^{34}$ 1) Arithmetic: The test researcher dictated the questions, and the subjects answered by mental arithmetic. Time limit was applied for each question. 
2) Digit span: Forward and backward number test, consisting of 11 questions, respectively. The forward number test started from 3 digits to 13 digits, while the backward number test started from 2 digits to 12 digits. The total score was the sum of the scores by forward and backward number test. 3) Coding: Each figure corresponded to a symbol. The subject was required to fill in the corresponding symbol in the lower part of the figure according to the specified figure-symbol relationship within a specified time.

\section{"Hot" executive function tests}

Iowa Gambling Task (IGT), which was designed by Bechara et al to mimic complex decision-making situations in real life, is considered as a classical experimental task for measuring emotional decision-making abilities, also a representative task to examine the "hot" executive function of ADHD patients. ${ }^{35}$ IGT was conducted before and 6 weeks after the treatment. The subjects were given 100 choices to randomly select one card from four sets of poker cards with back faced up on the screen. The face of each card showed the amount of reward or punishment. The card from the first and second sets of poker cards always indicated more rewards and more punishments, whereas the card from the third and fourth sets of poker cards always indicated less rewards and less punishments. Thus, the former cards were unfavorable, while the latter cards were favorable.

\section{Statistical analysis}

SPSS 17.0 was used for statistical analysis. Data were expressed as mean \pm standard deviation $(\bar{x} \pm s)$. Comparisons among groups were performed by using a repeated-measure ANOVA analysis test. Comparisons within groups were conducted by using a Paired $t$-test. If necessary, Chi-square test was used. All data except age were normally distributed. Pearson correlation analysis was used to assess the correlations between two variables. $P$-value $<0.05$ was considered as a statistical significance.

\section{Results}

\section{General demographic information}

A total of four patients dropped out during treatment, one from the rTMS group, two from the ATX group, and one from the combination group. The reasons were due to study stress, family economic conditions, and inconsistent parental opinions. The data from these patients were excluded. Thus, 60 ADHD patients were ultimately included into data analysis. These patients were aged from 6 to 13 years, with a mean age of $8.66 \pm 2.30$ years. There was no significant
Table I General demographics of the ADHD group

\begin{tabular}{l|l|l|l|l}
\hline & $\begin{array}{l}\text { rTMS } \\
(\mathbf{n}=\mathbf{2 0})\end{array}$ & $\begin{array}{l}\text { ATX } \\
(\mathbf{n}=1 \mathbf{1 9})\end{array}$ & $\begin{array}{l}\text { rTMS+ ATX } \\
(\mathbf{n}=\mathbf{2} \mathbf{)})\end{array}$ & P-value \\
\hline Age (years) & $8.36 \pm 2.46$ & $9.22 \pm 2.39$ & $8.50 \pm 2.20$ & $0.690^{*}$ \\
Sex (M/F) & $18 / 2$ & $16 / 3$ & $15 / 6$ & $0.289^{*}$ \\
\hline
\end{tabular}

Notes: Age is presented as mean \pm SD. *A repeated-measure ANOVA analysis or "chi-square test was used.

Abbreviations: ADHD, attention-deficit hyperactivity disorder; ATX, atomoxetine; rTMS, repetitive transcranial magnetic stimulation.

difference in age and gender among the rTMS group, the ATX group, and the combination group ( $P>0.05$; Table 1$)$. Finally, apart from mild scalp discomfort and headache, we did not observe any significant side effects.

\section{Clinical assessment results from SNAP-IV questionnaire}

A repeated-measure ANOVA analysis showed that there was a significant interaction effect in attention deficit, hyperactive impulse, and oppositional defiance in between treatment and time (all $P<0.001$ ). As shown in Table 2, the SNAP-IV questionnaire scores were statistically significant in attention deficit, hyperactivity impulsivity, and oppositional defiance disorder $(P<0.05)$ before and after treatment in the rTMS group, the ATX group, and the rTMS+ ATX group. By comparing the difference of the score before and after treatment for each item, the rTMS+ ATX group had greater improvements in attention deficit and hyperactivity impulsivity, but not in oppositional defiance, when compared with the other two groups (Figure 1).

\section{Assessments on "cold" and "hot" executive functions}

Significant time and treatment interaction effects were observed in arithmetic $(P<0.001)$, forward number $(P<0.01)$, backward number $(P<0.01)$, coding $(P<0.001)$, CPT $(P<0.001)$, IGT $(P<0.001)$ by a repeated-measure ANOVA analysis. There were statistically significant differences in the number of correct items from arithmetic test, and the scores from digit span test (including forward and backward number test, and their sum), as well as the scores from coding test between before and after treatment in the rTMS group, the ATX group, and the combination group (Table 3). Statistical significances were observed in the total score of CPT (calculated based on DEPRIME of each subtest) between before and after treatment (Table 3) in the three groups. The above results suggested that the three treatments were effective. Moreover, when compared with the rTMS group and the ATX group, the combination therapy exhibited 
Table 2 The scores of SNAP-IV questionnaire before and after treatment in the rTMS, the ATX, and the rTMS/ATX groups

\begin{tabular}{l|l|l|l|l|l|l}
\hline Factor & Group & $\mathbf{n}$ & BT & AT & t-value & $P$-value \\
\hline Attention deficit & rTMS & 20 & $2.13 \pm 0.35$ & $1.02 \pm 0.16$ & 14.54 & 0.000 \\
& ATX & 19 & $2.15 \pm 0.44$ & $1.08 \pm 0.15$ & 8.87 & 0.000 \\
Hyperactivity impulse & rTMS+ ATX & 21 & $2.18 \pm 0.36$ & $0.76 \pm 0.23$ & 15.54 & 0.000 \\
& rTMS & 20 & $1.91 \pm 0.45$ & $0.99 \pm 0.20$ & 10.41 & 0.000 \\
Oppositional defiance & ATX & 19 & $1.47 \pm 0.63$ & $0.64 \pm 0.24$ & 5.99 & 0.000 \\
& rTMS+ ATX & 21 & $1.79 \pm 0.62$ & $0.46 \pm 0.33$ & 12.82 & 0.000 \\
& rTMS & 20 & $1.07 \pm 0.57$ & $0.48 \pm 0.17$ & 4.60 & 0.001 \\
& ATX & 19 & $1.13 \pm 0.49$ & $0.51 \pm 0.25$ & 6.65 & 0.000 \\
& rTMS+ ATX & 21 & $1.21 \pm 0.39$ & $0.48 \pm 0.18$ & 9.15 & \\
\hline
\end{tabular}

Notes: Data are presented as mean \pm SD. Paired $t$-test was used in the statistical analysis.

Abbreviations: AT, after treatment; ATX, atomoxetine; BT, before treatment; rTMS, repetitive transcranial magnetic stimulation; SNAP-IV, the Swanson, Nolan, and Pelham, Version IV

a better effect on forward/backward number repeat and CPT, but not arithmetic and coding (Figure 2). Finally, in the three groups, there was a statistically significant difference in the score of IGT between before and after treatment. Relative to the rTMS group and the ATX group, the combination group had a greater improvement.

\section{Correlations between general characteristics and clinical symptoms and executive functions}

As shown in Table 4, age was negatively correlated with the score of attention deficits $(r=-0.36, P<0.05)$ and hyperactivity impulse $(-0.59, P<0.01)$, and was positively correlated with the score of CPT $(r=0.50, P<0.01)$, arithmetic ( $r=0.84, P<0.01)$, digit span $(r=0.67, P<0.01)$, and coding $(r=0.87, P<0.01)$.

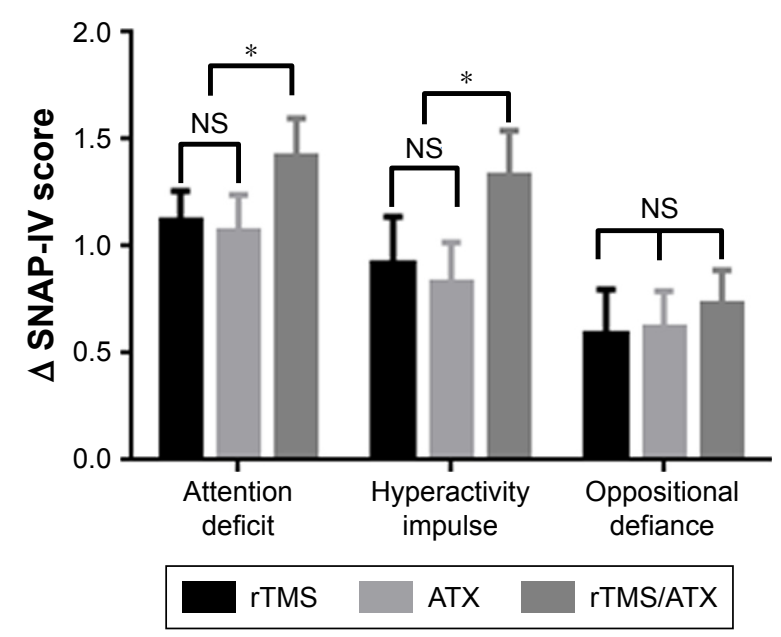

Figure I The differences of SNAP-IV scores before and after treatment in the rTMS, the ATX, and the rTMS/ATX groups.

Note: $* P<0.05$ compared to the $r T M S$ and the ATX groups, a repeated measure ANOVA was used.

Abbreviations: ATX, atomoxetine; NS, nonsignificant; rTMS, repetitive transcranial magnetic stimulation; SNAP-IV, the Swanson, Nolan, and Pelham, Version IV.
The score of attention deficits was negatively correlated with the score of CPT ( $r=-0.37, P<0.05)$, arithmetic ( $r=-0.49, P<0.01)$, digit span $(r=-0.36, P<0.05)$, and coding $(r=-0.40, P<0.05)$. The score of hyperactivity impulse was also negatively correlated with the score of CPT $(r=-0.61$, $P<0.01)$, arithmetic $(r=-0.66, P<0.01)$, digit span $(r=-0.45$, $P<0.01)$, and coding $(r=-0.58, P<0.01$; Table 5). However, there was no significant correlation between the score of hyperactivity impulse and the score of IGT (reflecting "hot" executive function; $r=0.029, P>0.05$ ). These results suggested that the core symptoms of ADHD are associated with cognitive functions.

\section{Discussion}

1. The rTMS therapy combined with ATX administration has the advantage over the single treatment in improving the core symptoms of ADHD.

Previous studies showed that the therapeutic effect of ATX for ADHD is significant, safe, and reliable by binding to the NE reuptake inhibitors on the presynaptic membrane to increase the concentration of NE in the synaptic gap. ${ }^{36,37}$ Consistently, in this study, we observed that ATX had a significant improvement on the three core symptoms (attention deficits, hyperactivity/impulsivity, and oppositional defiance) of ADHD after 6 weeks administration.

Previous studies used low-frequency or highfrequency rTMS with different parameters, and showed improvement in the patient's symptoms, such as attention deficit and hyperactivity. ${ }^{25,38,39}$ Specifically, Niederhofer used low-frequency rTMS $(1 \mathrm{~Hz})$ to stimulate the motor area (1,200 pulses per session, 5 sessions per week [from Monday to Friday]). After 4 weeks of treatment, ADHD symptoms were significantly improved. ${ }^{38}$ Weaver et al delivered high-frequency rTMS $(10 \mathrm{~Hz})$ 
Table 3 The scores of cold and hot executive functions before and after the three treatments

\begin{tabular}{l|l|l|l|l|l|l}
\hline Factor & Group & $\mathbf{n}$ & $\mathbf{B T}$ & AT & $\boldsymbol{t}$-value & $\boldsymbol{P}$-value \\
\hline Arithmetic & rTMS & 20 & $11.45 \pm 4.50$ & $12.18 \pm 4.83$ & 2.67 & 0.024 \\
& ATX & 19 & $12.22 \pm 6.52$ & $13.11 \pm 6.23$ & 8.00 & 0.000 \\
Forward number & rTMS+ ATX & 21 & $10.50 \pm 5.07$ & $11.75 \pm 4.71$ & 6.97 & 0.000 \\
& rTMS & 20 & $5.64 \pm 2.06$ & $6.27 \pm 1.90$ & 4.18 & 0.002 \\
Backward number & ATX & 19 & $6.11 \pm 2.09$ & $6.67 \pm 2.06$ & 3.16 & 0.013 \\
& rTMS+ ATX & 21 & $5.17 \pm 2.04$ & $6.32 \pm 1.68$ & 4.18 & 0.002 \\
Coding & rTMS & 20 & $3.91 \pm 1.04$ & $4.36 \pm 1.03$ & 2.89 & 0.016 \\
& ATX & 19 & $3.89 \pm 0.93$ & $4.56 \pm 0.73$ & 4.00 & 0.004 \\
CPT & rTMS+ ATX & 21 & $3.75 \pm 0.87$ & $4.33 \pm 0.99$ & 3.92 & 0.002 \\
& rTMS & 20 & $39.45 \pm 15.84$ & $42.36 \pm 16.39$ & 4.77 & 0.001 \\
IGT & ATX & 19 & $45.67 \pm 19.58$ & $49.11 \pm 19.42$ & 7.26 & 0.000 \\
& rTMS+ ATX & 21 & $37.42 \pm 15.56$ & $41.58 \pm 16.27$ & 11.39 & 0.000 \\
& rTMS & 20 & $0.87 \pm 0.34$ & $1.65 \pm 0.39$ & 8.94 & 0.001 \\
& ATX & 19 & $1.07 \pm 0.51$ & $1.79 \pm 0.28$ & 4.61 & 0.002 \\
& rTMS+ ATX & 21 & $0.88 \pm 0.60$ & $1.96 \pm 0.49$ & 12.97 & 0.000 \\
& rTMS & 20 & $-7.64 \pm 8.04$ & $4.45 \pm 8.26$ & 4.93 & 0.001 \\
& ATX & 19 & $-10.44 \pm 7.29$ & $7.44 \pm 2.96$ & 5.79 & 0.000 \\
& rTMS+ ATX & 21 & $-11.17 \pm 6.06$ & $10.58 \pm 7.89$ & 9.02 & 0.000 \\
\hline
\end{tabular}

Notes: Data are presented as mean \pm SD. Paired $t$-test was used.

Abbreviations: AT, after treatment; ATX, atomoxetine; BT, before treatment; CPT, continuous performance test; IGT, lowa Gambling Tasks; rTMS, repetitive transcranial magnetic stimulation.

to stimulate rDLPFC at 100\% motor threshold, 2,000 pulses per session, 5 sessions per week. They observed that the scores of CGI-I and ADHD-IV were significantly improved after 2 weeks of treatment. ${ }^{25}$ Consistently, our results showed that application of high-frequency rTMS over rDLPFC significantly improved attention deficits, hyperactivity impulse, and oppositional defiance. Based on these results, whether low-frequency rTMS over the motor area or high-frequency rTMS over rDLPFC can achieve a therapeutic effect, in addition to rTMS

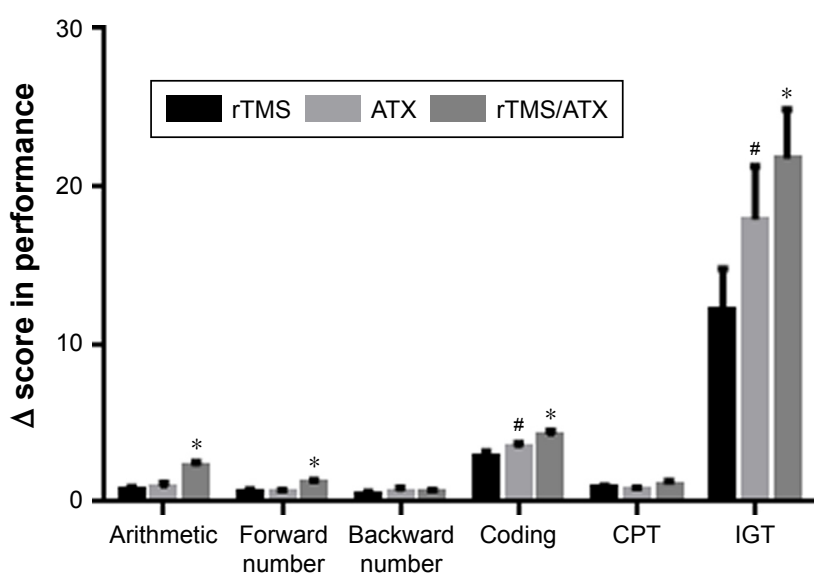

Figure 2 The differences of scores on cold and hot executive functions before and after treatment in the rTMS, the ATX, and the rTMS/ATX groups.

Notes: $* P<0.05$, compared to the rTMS group, and the ATX group; ${ }^{*} P<0.05$, compared to the rTMS group; a repeated measure ANOVA was used.

Abbreviations: ATX, atomoxetine; CPT, continuous performance test; IGT, lowa Gambling Task; rTMS, repetitive transcranial magnetic stimulation. regulating neuroplasticity, promoting the release of neurotransmitters and neurotrophic factors, and improving blood circulation, we speculated that these protocols share common effects of magnetic stimulation, such as quantum effect and magnetic spin effect, altering the atomic and molecular magnetic state of disorder in the stimulated brain. ${ }^{40}$ However, which protocol is more effective requires further investigation.

This study further investigated the effect of the rTMS therapy combined with ATX for ADHD, and showed that combination therapy was also effective, and had a greater improvement in two core symptoms, attention deficit and hyperactivity impulse. There are few studies on the combined treatment of ADHD with two different methods. It has been reported that ATX or rTMS in combination with the behavior training had a better therapeutic effect when compared with the single method, but there are some reports showing no obvious advantage..$^{41,42}$ However, further in-depth research may need to increase sample size and optimize the treatment parameters.

2. The rTMS therapy combined with ATX administration has the advantage over the single treatment in improving executive functions of ADHD.

This study showed that "cold" and "hot" executive functions of ADHD patients were impaired, reflecting their impaired prefrontal function. The impairment of 
Table 4 Correlation coefficient between age and attention deficit, hyperactivity impulse, executive function $(r)$

\begin{tabular}{l|l|l|l|l|l|l}
\hline & $\begin{array}{l}\text { SNAP-IV } \\
\text { Attention deficit }\end{array}$ & $\begin{array}{l}\text { SNAP-IV } \\
\text { Hyperactivity impulse }\end{array}$ & CPT DEPRIME & Arithmetic & Coding & Digit span \\
\hline Age & $-0.36 *$ & $-0.59 *$ & $0.50^{* *}$ & $0.84^{* *}$ & $0.87^{* *}$ & $0.67^{* *}$ \\
\hline
\end{tabular}

Notes: $* P<0.05$. $* * P<0.01$. Pearson correlation analysis was used.

Abbreviations: SNAP-IV, the Swanson, Nolan, and Pelham, Version IV; CPT, continuous performance test.

"cold" executive function is reflected in the attention deficit, while the "hot" executive function is closely related to impulse behavior. ${ }^{29}$ In this study, the three treatment approaches were effective in improving cold and hot executive functions. Also, there was a significant difference in the scores of arithmetic, forward number repeat, coding, and IGT in the combined group when compared with the rTMS group or the ATX group, suggesting that the combination therapy has some advantages over the single treatment in improving attention maintenance and decision-making ability of ADHD patients. Interestingly, ATX therapy was superior to rTMS in coding and IGT; this might be because our rTMS protocol is not optimal.

Previous studies suggested that children with ADHD have decision-making problems in real life, which are difficult to correct through learning and will last for a long time ${ }^{43}$ In this study, almost all patients had a score of IGT $<0$ before treatment, suggesting that they were more inclined to choose unfavorable cards with immediate higher reward and higher punishment, and failed to explore intrinsic rules in the process of operation and to learn ways to increase income. There are two possible explanations. First, ADHD patients paid attention only to immediate short-term benefits, regardless of long-term benefits; second, even if the long-term interests were considered, they still could not restrain their preference for current high reward. This was closely related to the defects in their work memory, impulsive inhibition, and logical reasoning. There is evidence that the ventral prefrontal cortex, which is not mature until puberty, is involved in judgment of long-term interests. ${ }^{44}$ Therefore, there is a certain objective factor in the decision-making ability of children with ADHD.
3. Correlation analysis of general information, clinical symptoms, and executive functions.

The correlation analysis between age and clinical symptoms and executive functions suggested that the ability to maintain attention and control hyperactivity/ impulsivity might increase with age in adolescents, and that the brain regions involved in audiovisual work memory and attention maintenance (such as dorsolateral prefrontal lobe) might mature gradually. Arithmetic and digit span test were used to examine the level of auditory attention and auditory working memory, ${ }^{34,45}$ while CPT and coding test were used to examine the level of visual attention and visual work memory. ${ }^{46,47}$ Therefore, attention and work memory play a role in these tests. The negative correlation of the score of attention deficit with the total score of CPT, arithmetic, digit span, and coding implicated that greater ability in attention and maintenance might lead to a better performance in executive function tests. Our results were consistent with the notion that attention deficit was related to the "cold" executive functions proposed by Castellanos et al. ${ }^{29}$

The negative correlations between hyperactive impulsivity and cold executive functions suggested that hyperactive impulsivity was highly related to work memory. If ADHD patients can improve their impulse control, their attention level and attention maintenance ability will increase correspondingly, leading to a higher level of focused attention in collecting effective information. On the basis of sustained attention, the efficiency of processing and transforming information (namely working memory) will also improve correspondingly. ${ }^{48-50}$ These also suggest that there are internal interactions between the core symptoms of ADHD. Thus, clinicians

Table 5 Correlation coefficient between attention deficit, hyperactivity impulse and CPT, arithmetic, coding, digit span ( $r$ )

\begin{tabular}{l|l|l|l|l}
\hline SNAP-IV & CPT DEPRIME & Arithmetic & Coding \\
\hline Attention deficit & $-0.37^{*}$ & $-0.49 * *$ & $-0.40^{*}$ & Digit span \\
Hyperactivity impulse & $-0.6 I^{* *}$ & $-0.66^{* *}$ & $-0.58^{* *}$ & $-0.36^{*}$ \\
\hline
\end{tabular}

Notes: $* P<0.05, * * P<0.01$. Pearson correlation analysis was used.

Abbreviation: $\mathrm{CPT}$, continuous performance test. 
cannot separate attention deficit and hyperkinetic impulse at the diagnosis of ADHD.

IGT, as a classic test to examine "hot" executive function, reflects the individual ability of emotional decision-making, and is related to hyperactivity impulse symptom. However, in this study, it appeared that the score of hyperactivity impulse was not significantly correlated with the IGT score. There are several possible reasons. First, ADHD patients aged from 6 to 13 years, had a relatively scattered distribution. Thus, there were differences in the development and maturity of related brain regions. There is evidence that even in normal adults a significant proportion of persons cannot perform well in IGT. Bechara et al found that about 33\%-37\% of normal adults had comparable IGT scores to patients with the injury of ventromedial prefrontal lobe. ${ }^{35}$ Second, there were differences in the criteria of parents for judging the severity of children symptoms. Some parents thought that their children were just more active than others; some parents felt that their children were very quiet when watching TV or using computer. Barkley et al found that there was no significant difference in impulse control and working memory for relatively interesting tasks between ADHD children and normal children. But for relatively boring tasks, the difference between the two groups was significant. ${ }^{51,52}$ Third, individual difference and the emotional status in ADHD patients during test may also affect the outcome. Buelow and Suhr observed that college students with negative emotions, impulsiveness, or thrill-seeking were more inclined to choose unfavorable cards with more rewards and more punishments, suggesting that emotions and personality characteristics have a great impact on risk decision-making. ${ }^{53}$

\section{Limitations}

This study still has several limitations. First, the sample size was small; thus larger samples are needed to validate the results. Second, the combination therapy needs to be further optimized. The doses of ATX and treatment parameters of rTMS need to be individualized. How to combine the two treatments and how to adjust the time of medication and physical therapy for maximizing the efficacy is one direction of future research. Third, this study failed to set the placebo group and sham rTMS group, thus the placebo effects could be not excluded. Fourth, although this was relatively a longer therapy protocol, it is unclear whether a longer therapy (3 months, even 6 months) achieves greater synergistic effects. The ATX administration is convenient, but it may not be easy for patients to visit hospitals frequently for rTMS therapy. Finally, we failed to perform follow-up, lacking the observation of long-term effect of the treatment.

\section{Conclusion}

Our results showed that rTMS, ATX, or rTMS combined with ATX can effectively alleviate attention deficit, hyperactivity impulse, and oppositional defiance in ADHD children, and can also improve executive functions including working memory, inhibitory control, and emotional decision-making. When compared with single treatment, rTMS combined with ATX showed significant advantages in attention deficit and hyperactivity impulse, as well as in "cold" and "hot" executive functions such as arithmetic, forward numbers, coding, and IGT. The ATX drug treatment was superior to rTMS treatment in coding and IGT. In addition, age was negatively correlated with the score of attention deficit and hyperactivity impulse, and was positively correlated with the score of "cold" executive functions; the score of attention deficit was negatively correlated with the score of "cold" executive functions, whereas the score of hyperactivity impulse has no statistically significant correlation with the score of "hot" executive function. In summary, the three treatments have definite efficacy in ADHD, and the combined treatment has some significant advantages over single treatments. How to optimize the combination therapy to achieve better therapeutic effect is the future direction of investigation.

\section{Data statement}

The authors intend to share all individual deidentified participant data except patients' privacy. Under the request, the authors will send related data by email. These data will be available from publication to 5 years after publication.

\section{Acknowledgments}

This work was funded by National Natural Science Foundation of China (NOs 81471338 and 81671284 to XZ), Changzhou Science and Technology Program Grant (CE20145045 to XZ), and the Changzhou High-Level Medical Talents Training Project (NO 2016CZLJ018 to XZ).

\section{Author contributions}

$\mathrm{XZ}$ and $\mathrm{ZS}$ conceived and designed this study protocol. $\mathrm{PC}$, JX, YC, QC, XS, QK, and LD performed this study. XZ, ZS, $\mathrm{PC}$, and JX discussed and conducted data analysis. XZ and PC wrote this manuscript. All authors contributed to data analysis, drafting or revising the article, gave final approval of the version to be published, and agree to be accountable for all aspects of the work. 


\section{Disclosure}

The authors report no conflicts of interest in this work.

\section{References}

1. Association AP. Diagnostic and Statistical Manual of Mental Disorders: DSM-5. Arlington, VA: American Psychiatric Association; 2013:947.

2. Thapar A, Cooper M, Eyre O, Langley K. Practitioner review: what have we learnt about the causes of ADHD? J Child Psychol Psychiatry. 2013;54(1):3-16.

3. Pingault JB, Viding E, Galéra C, et al. Genetic and environmental influences on the developmental course of attention-deficit/hyperactivity disorder symptoms from childhood to adolescence. JAMA Psychiatry. 2015;72(7):651-658.

4. Gornick MC, Addington A, Shaw P, et al. Association of the dopamine receptor D4 (DRD4) gene 7-repeat allele with children with attentiondeficit/hyperactivity disorder (ADHD): an update. Am J Med Genet B Neuropsychiatr Genet. 2007;144B(3):379-382.

5. Rubia K, Smith AB, Brammer MJ, Taylor E. Temporal lobe dysfunction in medication-naïve boys with attention-deficit/hyperactivity disorder during attention allocation and its relation to response variability. Biol Psychiatry. 2007;62(9):999-1006.

6. Gilliam M, Stockman M, Malek M, et al. Developmental trajectories of the corpus callosum in attention-deficit/hyperactivity disorder. Biol Psychiatry. 2011;69(9):839-846.

7. Smith AB, Taylor E, Brammer M, Toone B, Rubia K. Task-specific hypoactivation in prefrontal and temporoparietal brain regions during motor inhibition and task switching in medication-naive children and adolescents with attention deficit hyperactivity disorder. Am J Psychiatry. 2006;163(6):1044-1051.

8. Shaw P, Malek M, Watson B, Sharp W, Evans A, Greenstein D. Development of cortical surface area and gyrification in attention-deficit/ hyperactivity disorder. Biol Psychiatry. 2012;72(3):191-197.

9. Lou HC, Henriksen L, Bruhn P. Focal cerebral dysfunction in developmental learning disabilities. Lancet. 1990;335(8680):8-11.

10. Zametkin AJ, Nordahl TE, Gross M, et al. Cerebral glucose metabolism in adults with hyperactivity of childhood onset. $N$ Engl J Med. 1990; 323(20):1361-1366.

11. Banerjee TD, Middleton F, Faraone SV. Environmental risk factors for attention-deficit hyperactivity disorder. Acta Paediatr. 2007;96(9): 1269-1274.

12. Silva D, Colvin L, Hagemann E, Bower C. Environmental risk factors by gender associated with attention-deficit/hyperactivity disorder. Pediatrics. 2014;133(1):e14-e22.

13. Thapar A, Cooper M. Attention deficit hyperactivity disorder. Lancet. 2016;387(10024):P1240-P1250.

14. Roskell NS, Setyawan J, Zimovetz EA, Hodgkins P. Systematic evidence synthesis of treatments for ADHD in children and adolescents: indirect treatment comparisons of lisdexamfetamine with methylphenidate and atomoxetine. Curr Med Res Opin. 2014;30(8):1673-1685.

15. Adler LA, Spencer TJ, Williams DW, Moore RJ, Michelson D. Longterm, open-label safety and efficacy of atomoxetine in adults with ADHD: final report of a 4-year study. J Atten Disord. 2008;12(3):248-253.

16. Michelson D, Faries D, Wernicke J, et al. Atomoxetine in the treatment of children and adolescents with attention-deficit/hyperactivity disorder: a randomized, placebo-controlled, dose-response study. Pediatrics. 2001;108(5):E83.

17. Upadhyaya H, Kratochvil C, Ghuman J, et al. Efficacy and safety extrapolation analyses for atomoxetine in young children with attentiondeficit/hyperactivity disorder. J Child Adolesc Psychopharmacol. 2015; 25(10):799-809.

18. Barker AT, Jalinous R, Freeston IL. Non-invasive magnetic stimulation of human motor cortex. Lancet. 1985;1(8437):1106-1107.
19. Balconi M, Ferrari C. Left DLPFC rTMS stimulation reduced the anxiety bias effect or how to restore the positive memory processing in high-anxiety subjects. Psychiatry Res. 2013;209(3):554-559.

20. Fox MD, Liu H, Pascual-Leone A. Identification of reproducible individualized targets for treatment of depression with TMS based on intrinsic connectivity. Neuroimage. 2013;66(66):151-160.

21. Rabany L, Deutsch L, Levkovitz Y. Double-blind, randomized sham controlled study of deep-TMS add-on treatment for negative symptoms and cognitive deficits in schizophrenia. J Psychopharmacol. 2014;28(7): 686-690.

22. Rossi S, Hallett M, Rossini PM, Pascual-Leone A. Safety of TMS Consensus Group. Safety, ethical considerations, and application guidelines for the use of transcranial magnetic stimulation in clinical practice and research. Clin Neurophysiol. 2009;120(12):2008-2039.

23. Bloch Y, Harel EV, Aviram S, Govezensky J, Ratzoni G, Levkovitz Y. Positive effects of repetitive transcranial magnetic stimulation on attention in ADHD Subjects: a randomized controlled pilot study. World $J$ Biol Psychiatry. 2010;11(5):755-758.

24. Lefaucheur JP, André-Obadia N, Antal A, et al. Evidence-based guidelines on the therapeutic use of repetitive transcranial magnetic stimulation (rTMS). Clin Neurophysiol. 2014;125(11):2150-2206.

25. Weaver L, Rostain AL, Mace W, et al. Transcranial magnetic stimulation (TMS) in the treatment of attention-deficit/hyperactivity disorder in adolescents and young adults. J ECT. 2012;28(2):98-103.

26. Swanson JM, et al. The SNAP Rating Scale for the diagnosis of the attention deficit disorder. Paper presented at the Annual Meeting of the American Psychology Association; November 18, 1981; Washington DC.

27. Swanson JM, Kraemer HC, Hinshaw SP, et al. Clinical relevance of the primary findings of the MTA: success rates based on severity of ADHD and ODD symptoms at the end of treatment. J Am Acad Child Adolesc Psychiatry. 2001;40(2):168-179.

28. Collett BR, Ohan JL, Myers KM. Ten-year review of rating scales. $\mathrm{V}$ : scales assessing attention-deficit/hyperactivity disorder. J Am Acad Child Adolesc Psychiatry. 2003;42(9):1015-1037.

29. Castellanos FX, Sonuga-Barke EJ, Milham MP, Tannock R. Characterizing cognition in ADHD: beyond executive dysfunction. Trends Cogn Sci. 2006;10(3):117-123.

30. Skogli EW, Andersen PN, Hovik KT, Øie M. Development of hot and cold executive function in boys and girls with ADHD. J Atten Disord. 2017;21(4):305-315.

31. Zhu Y, Jiang X, Ji W. The mechanism of cortico-striato-thalamo-cortical neurocircuitry in response inhibition and emotional responding in attention deficit hyperactivity disorder with comorbid disruptive behavior disorder. Neurosci Bull. 2018;34(3):566-572.

32. Antonini TN, Becker SP, Tamm L, Epstein JN. Hot and cool executive functions in children with attention-deficit/hyperactivity disorder and comorbid oppositional defiant disorder. J Int Neuropsychol Soc. 2015; 21(8):584-595.

33. Yu X. MCCB Chinese Norm Manual. China: Peking University Medical Press; 2014.

34. Fried R, Chan J, Feinberg L, et al. Clinical correlates of working memory deficits in youth with and without ADHD: a controlled study. J Clin Exp Neuropsychol. 2016;38(5):487-496.

35. Bechara A, Damasio H, Damasio AR, Lee GP. Different contributions of the human amygdala and ventromedial prefrontal cortex to decisionmaking. J Neurosci. 1999;19(13):5473-5481.

36. Kratochvil CJ, Vaughan BS, Stoner JA, et al. A double-blind, placebocontrolled study of atomoxetine in young children with ADHD. Pediatrics. 2011;127(4):e862-e868.

37. Michelson D, Buitelaar JK, Danckaerts M, et al. Relapse prevention in pediatric patients with ADHD treated with atomoxetine: a randomized, double-blind, placebo-controlled study. J Am Acad Child Adolesc Psychiatry. 2004;43(7):896-904.

38. Niederhofer H. Effectiveness of the repetitive transcranial magnetic stimulation (rTMS) of $1 \mathrm{~Hz}$ for attention-deficit hyperactivity disorder (ADHD). Psychiatr Danub. 2008;20(1):91-92. 
39. Niederhofer H. Additional biological therapies for attention-deficit hyperactivity disorder: repetitive transcranial magnetic stimulation of $1 \mathrm{~Hz}$ helps to reduce methylphenidate. Clin Pract. 2012;2(1):e8.

40. Chervyakov AV, Chernyavsky AY, Sinitsyn DO, Piradov MA. Possible mechanisms underlying the therapeutic effects of transcranial magnetic stimulation. Front Hum Neurosci. 2015;9(9):303.

41. Smith T, Aman MG, Arnold LE, et al. Atomoxetine and parent training for children with autism and attention-deficit/hyperactivity disorder: a 24-week extension study. J Am Acad Child Adolesc Psychiatry. 2016; 55(10):868-876.

42. Arnold LE, Ober N, Aman MG, et al. A 1.5-year follow-up of parent training and atomoxetine for attention-deficit/hyperactivity disorder symptoms and noncompliant/disruptive behavior in autism. J Child Adolesc Psychopharmacol. 2018;28(5):322-330.

43. Hawthorne MJ, Pierce BH. Disadvantageous deck selection in the Iowa Gambling Task: the effect of cognitive load. Eur J Psychol. 2015;11(2): 335-348.

44. Li X, Lu ZL, D’Argembeau A, Ng M, Bechara A. The Iowa Gambling Task in fMRI images. Hum Brain Mapp. 2010;31(3):410-423.

45. Wells EL, Kofler MJ, Soto EF, Schaefer HS, Sarver DE. Assessing working memory in children with ADHD: minor administration and scoring changes may improve digit span backward's construct validity. Res Dev Disabil. 2018;72(72):166-178.

46. Berger I, Slobodin O, Cassuto H. Usefulness and validity of continuous performance tests in the diagnosis of attention-deficit hyperactivity disorder children. Arch Clin Neuropsychol. 2017;32(1):81-93.
47. Nelwan M, Vissers C, Kroesbergen EH. Coaching positively influences the effects of working memory training on visual working memory as well as mathematical ability. Neuropsychologia. 2018;113(113): $140-149$.

48. Friedman LM, Rapport MD, Raiker JS, Orban SA, Eckrich SJ. Reading comprehension in boys with ADHD: the mediating roles of working memory and orthographic conversion. J Abnorm Child Psychol. 2017; 45(2):273-287.

49. Patros CHG, Alderson RM, Hudec KL, Tarle SJ, Lea SE. Hyperactivity in boys with attention-deficit/hyperactivity disorder: the influence of underlying visuospatial working memory and self-control processes. J Exp Child Psychol. 2017;154:1-12.

50. Kennedy RJ, Quinlan DM, Brown TE. Comparison of two measures of working memory impairments in 220 adolescents and adults with ADHD. J Atten Disord. Epub 2016 Aug 1.

51. Barkley RA, Fischer M. Predicting impairment in major life activities and occupational functioning in hyperactive children as adults: self-reported executive function (EF) deficits versus EF tests. Dev Neuropsychol. 2011; 36(2):137-161.

52. Barkley RA, Edwards G, Laneri M, Fletcher K, Metevia L. Executive functioning, temporal discounting, and sense of time in adolescents with attention deficit hyperactivity disorder (ADHD) and oppositional defiant disorder (ODD). J Abnorm Child Psychol. 2001;29(6):541-556.

53. Buelow MT, Suhr JA. Personality characteristics and state mood influence individual deck selections on the Iowa Gambling Task. Pers Individ Dif. 2013;54(5):593-597.
Neuropsychiatric Disease and Treatment

\section{Publish your work in this journal}

Neuropsychiatric Disease and Treatment is an international, peerreviewed journal of clinical therapeutics and pharmacology focusing on concise rapid reporting of clinical or pre-clinical studies on a range of neuropsychiatric and neurological disorders. This journal is indexed on PubMed Central, the 'PsycINFO' database and CAS,

\section{Dovepress}

and is the official journal of The International Neuropsychiatric Association (INA). The manuscript management system is completely online and includes a very quick and fair peer-review system, which is all easy to use. Visit http://www.dovepress.com/testimonials.php to read real quotes from published authors. 